\title{
DOCUMENTOS
}




\section{O TRATADO DE CANGUÊ}

Em 1. ${ }^{\circ}$ de março de 1835, Manuel Oribe foi eleito presidente da República Oriental do Uruguai, não havendo outro candidato e nem oposição à sua ascensão ao poder. Fructuoso Rivera, que fora presidente de 1830 a 1835 , desempenhava a função de Comandante Geral da Campanha, com amplíssimos poderes sobre chefes militares e eleições de alcaides, gerando conflitos com o governo de Oribe em Montevidéu. O decreto de 9 de janeiro de 1836 suprimiu a Comandância Geral da Campanha, numa tentativa de unificar o poder. Iniciou-se também um processo financeiro sobre a precedente administração de Rivera.

Em 18 de julho de 1836 Rivera alçou-se em armas, com auxílio de imigrados políticos argentinos. Buscou ainda auxílio dos farroupilhas, que desde 20 de setembro de 1835, estavam em armas na província do Rio Grande do Sul contra o Império do Brasil.

Em 21 de agosto de 1838, Fructuoso Rivera assinou o tratado de Canguê, com a República Rio-Grandense, formando uma aliança ofensiva-defensiva contra os inimigos comuns. A finalidade básica deste tratado era de que os farroupilhas reconhecessem Rivera como futuro presidente do Uruguai. Aos sul-rio-grandenses interessava o caminho livre para a fronteira, a fim de refazerem suas tropas militares ou conseguir armas e munições. $\mathrm{O}$ tratado nunca chegou a ser executado porque tanto os farroupilhas como Rivera fizeram jogo duplo, aqueles ora apoiando Oribe, ora Rivera; e este fingindo apoiar os farroupilhas, enquanto recebia dinheiro do Império do Brasil para combatê-los.

A cópia do Tratado de Canguê encontra-se no livro de registro da Secretaria de Estado dos Negócios do Exterior, da República Rio-Grandense, na coleção de Alfredo Varela, do Arquivo Histórico do Rio Grande do Sul. 
El Presidente de la Republica Rio Grandense y el General en Jefe Defensor de la Constitucion de la Republica Oriental del Uruguay, animados de un sincero de ceo de restituir la paz y la tranquilidad interna a cada uno de los respectivos Estados, fuertemente alteradas por la resistencia legal de los Pueblos contra sus implacables inemigos y opressores, los Gobiernos que en otra hora los administravam, y no encontrando medio mas obvio y natural para la consecusion del fim propuesto que el de unirse en virtud de un tratado de Alianza Ofensiva y defensiva que reconecerá como bases las estipulaciones consagradas en los articulos abajo referidos de la presente Convencion Preliminar bien como servirar para el mismo fin y efecto aquelos otros de la ya celebrada convencion amigable entre las mismas Altas Partes Contratantes, datada em diez de Junio de este ano en la Ciudad de Piratiny e que tendrá el mismo valor $\mathrm{y}$ efecto de un tratado regular y en forma revistido de todas suas solemnidades para este objecto convinicron las dos altas partes contratantes en nombrar por seus Plenipotenciarios a saber $=$ Su Exelencia el Senor Prezidente de la Republica Rio-Grandense al Senor Joze Mariano de Matos, Coronel Commandante del primer cuerpo de Artilleria a caballo; e Su Exelencia el Senor General en Jefe defensor de la Constitucion de la Republica Oriental á Don Andres Lamas, Auditor General de Guerra del Ejercito Constitucional y a Don Martiniano Chilavert Coronel de Artilleria del mismo Ejercito, los cuales despues de haber cangeado, examinado y encontrado en debida forma sus respectivos Plenos poderes convenieron en los articulos seguintes:

Articulo $1 .^{\circ}$ - El General Presidente de la Republica Rio-Grandense reconose en nombre, por y de parte de su Republica, en la persona del Excclentisimo Senor General en Jefe del Ejercito Constitusional Brigadier General Don Fructuozo Rivera la unica superior autoridad existente hoy en la Republica Oriental del Uruguay, por el voto de la Nacion reunida bajo sus ordenes para sostener sus instituciones: y conseguientemente se declara en estado de guerra contra todos los enemigos internos o esternos a la Causa que sustiene el mencionado General en Jefe desde que la presente convencion sea legal y difinitivamente ratificada por el Gobierno que establesca la República Oriental.

Articulo 2. ${ }^{\circ}$ - El General en Jefe del Ejercito Constitucional reconose en nombre de la Republica y del Ejercito Oriental la Independencia y el Título de la Republica Rio-Grandense; y se constituye en estado de guerra contra todos los enemigos internos ó esternos de la misma Republica desde que la presente convencion sea legal y definitivamente ratificada: quedando desde luego abierto los Puertos de la Republica para los buques patentados por el Gobierno Rio-Grandense.

Articulo $3 .^{\circ}$ - El General en Jefe del Ejercito Constitucional y los Jefes signatarios de la Ratificacion provisoria de esta Convencion, se obligan a obtener por su influencia la ratificacion legal y solemne del Gobierno que establesca la Republica despues del completo triunfo de la Causa Santa que defienden. Se obligan tambien a obtner por esa misma influensa la celebracion entre los Gobiernos de outras Republicas de un tratado ofensivo y defensivo contra sus comunes enemigos, dentro del plazo de cincuenta dias a contar despues de la ratificasion difinitiva de la presente Convencion.

Articulo $4 .^{\circ}$ - El mismo Senor General en Jefe immediatamente despues de la ratificasion de esta Convension acreditará cerca de la persona del Presidente de la Repubiica Rio-Grandense un Enviado Extraordinario provisto de Plenos Poderes para concluir cualquier ajuste, disposicion ó convencion que emprevistos acontesimientos de la guerra demandaren y que deban ser immediatamente aprovados sin demora ó lapso de tempo contrario a los intereses de la Alianza. Y reciprocamente el General Presidente de la Republica Rio-Grandense acreditará cerca del General Defensor un Ministro Rio-Grandense de igual caracter y para el supra dicho fin.

Articulo $5 .^{\circ}$ - Desde el momento en que fuera ratificado el presente Convenio por el Gobierno de la Republica como se estipula en el articulo tercero se prosederá por el mismo Gobierno a la espulsion del territorio Oriental de todos los Agentes politicos del Imperio que en el fueren encontrados: quedando Su Exelencia el General en Jefe obligado desde la ratificasion provisoria a mandar desarmar los grupos armados que el Gobicrno Imperial mantien en el 
territorio Oriental con entero desprecio de la Soberania Nacional asi ultrajada, haciendo entrega al Gobierno de la Republica Rio-Grandense del armamento, municiones y de mas propriedades publicas que a tales grupos se encontraren.

Articulo $6 .^{\circ}$ - La esecucion de las estipulaciones declaradas en el articulo anterior será aplicable mutatis mutandis al Prezidente de la Republica del Rio-Grandense a favor de la Causa que sustiene el General en Jefe del Ejercito Constitucional.

Articulo $70^{\circ}$ - Será permittido al General Presidente de la Republica Rio-Grandense siempre que la necessidad de bater y hostilisar las fuerzas Imperiales lo exija, el hacer atravesar por sus tropas en identicas sircunstancias, el territorio Rio Grandense $\infty$ n la clausula supra dicha.

Articulo $8 .^{\circ}$ - Para llenar una exijencia política, y para dar al enemigo comum la mas alta idea de la estrecha alianza en cuestion, y robustecer cada vez mas los lasos de indisolubre amistad y de perfecta intelegencia entre los Pueblos; immediatamente despues de publicada la presente Convencion, Su Exelencia el General Defensor, pondrá á dispozision de Su Exelencia, el Presidente de la Republica del Rio-Grande, un Escuadron del Ejercito Constitucional completamente armado con trez caballos por plaza el cual permanecerá unido en cualidad de Auziliar al Ejercito Rio-Grandense en cuanto durara la guerra de la Independencia de este paiz; quedando tambien obligado el Presidente de la Republica del Rio Grande á poner igual fuerza de la misma arma, y por el tiempo arriba fijado á disposicion del General Defensor desde el momiento en que lo requiera. Ulteriores determinaciones marcaran el modo en que seran provistos de vestuario y de sueldo los indicados contingentes.

Articulo $9 .^{\circ}$ - Se comprometen ambas las altas Partes contratantes a entender-se para el futuro entre si y amigablemente en todo cuanto fuere relativo á la linea divisoria á contestar entre los dos paises, no admitiendo en este negocio la intervencion, buenos oficios ó influencia de una Potencia estrana qualquiera.

Articulo 10 - El General Presidente se obliga en nombre de la Republica á prover al General Defensor, luego que haya redusido la Ciudad del Rio Grande y ocupado la barra de aquel Porto, de toda la cuantidad de polvora, balas, y de mas recursos y utensilios de que pueda entonces disponer: obligacion aque igualmente se compromete el General Defensor luego que se apodere de la Ciudad de Monte Video.

Articulo 11 - El General Defensor se obliga a poner à la disposicion de la Republica Rio-Grandense y en el menor tiempo que le sea posible, trez mil caballos para el uso de sua Caballaria de Linea; los cuales le seran pagos luego que las fuerzas Imperiales fueren espulsadas del Territorio de la Republica.

Articulo 12 - E1 General Defensor se obliga a hacer acantonar una fuerza en el punto de Santa Teresa con el fin de observar los movimientos de las fuerzas Imperiales, imperdirles el transito, vedarles por todos los medios a su alcanse que por aquella parte reciba el enemigo de la Republica Rio-Grande cualquier especie de auxilio de la Banda Oriental en ganado vacuno, cabalgar, ó de cualquier otra especie.

Articulo 13 - El General Presidente acantonará para fines identicos en el territorio Rio-Grandense y en el lugar que le fuere designado una fuerza proporcional sacada de su Ejercito.

Articulo 14 - Ambas las Altas partes contratantes se obligan a porseguir em sus respectivos territorios cualquier introducion clandestina de toda especie de propriedades publicas ó particulares prestando ademas la mas seria atencion, y la cooperacion mas eficaz a las declaraciones que se hagan sobre tales objectos y mui particularmente á las que versen sobre la propriedad de los esclavos que se asilen ó conduscan de uno a otro territorio, en todo aquello que uno lastime la Legislacion vigente sobre la materia en cada uno de los dos Estados.

Articulo 15 - El General Defensor queriendo dar a la Republica Rio-Grandense una prueva no equivoca de lo mucho que la considera conviene á solicitud de Su Exelencia el 
General Prezidente de la misma Republica en amnistiar a aquellos Ciudadanos Orientales que servieron hasta aqui á la causa Republicana Rio-Grandense: salvo perjuicio de tercero, y siempre que el individuo amnistiado no haya cometido en la actual lucha Oriental ninguno de aquellos crimines horrorosos cuyos perpetradores son escluidos por las Leyes de todos los Pueblos civilisados de su immediata protecion, y del direcho de gentes. Y Su Exelencia el Presidente de La Republica Rio-Grandense á pedimento del General en Jefe del Ejercito Constitucional convien en hacer participar del mismo beneficio e indulgencia a los subditos Brasileros amigos de la Causa Constitucional de la Republica Oriental, que no se encontrem en el caso de los exeptuados en el primer miembro de este articulo - Una Comision nombrada ad hoc por las Altas Partes Contratantes clasificaran y designaran los individuos de ambos paises que deban ser amnistiados.

Articulo 16 - La presente Convencion Preliminar se conservará inviolablemente secreta hasta despues de la ratificasion de que habla el articulo tercero.

Articulo 17 - La presente Convension será desde luego ratificada por el General Jefe, y por los Generales y Oficiales Superiores del Ejercito Constitucional; y dentro del plazo de quince dias a contar de su data lo será por el General Presidente y por los Generales e Oficiales superiores del Ejercito Rio-Grandense, y consecuentemente cange adas las ratificasiones para su debido efecto y valor.

Para firmesa de lo cual Nos los abajo firmados Plenipotenciarios de Sus Exelencias el General Presidente de la Republica Rio-Grandense y el General en Jefe Defensor de la Constitucion de la Republica Oriental, en virtud de nuestros Plenos Poderes, firmamos la presente Convencion Preliminar con nuestros punos, y le hisimos poner el sello que usamos. Fecha en el Cuartel General del Ejercito Constitucional en la margen esquierda del Cangue a los veinte y un dias del mez de Agosto de mil ocho sientos treinta y ocho anos. Andres Lamas= Martiniano Chillaver = Joze Marianno de Mattos = Nós Fructuoso Rivera, Brigadier Grãl. de la Republica Oriental del Uruguay y Grãl. en Jefe del Ejto. Constitucional, y de mas Jefes reunidos para imponerse de la Convencion preliminar de la paz acordada entre el Embíado Estraordinario de S. E. el S. Gral. Presidente de la Republica Rio-Grandense, Coronel de Artilleria Joze Mar, de Matos y los Comisionados del Ejto. Coronel D. Martiniano Chilabert y Auditor gr̃al del Est ${ }^{\mathrm{O}}$. D. Andres Lamas, bien examinado cuanto contiene la precitada Convencion preliminar, ratificamos los articulos que contiene en todas sus partes empenando nuestra palabra y fe publica para cumplir y hacerla cumplir por todos los medios que ven a nuestro alcances; y para mayor seguridad la firmamos, siendo refrendada por el Secretario gl. del Est ${ }^{\circ}$ en el Cuartel gr̃al. de Cangué a veinte y un dia del mez de Agosto de mil ocho sientos treinta e ocho anos $=$ Fructuoso Rivera $=$ Felix Ed.do Aguiar $=$ Pablo Peres $=$ Rufino Baucá $=$ Vicente Vinó $=$ Angelo Nunes $=$ Venan $^{\circ}$ Flores $=$ Luciano Blanco $=$ Furt unato Mieres $=$ Juan Santander $=$ Hipolito $\mathrm{C}$. Quadros $=$ Abor Or $\mathrm{O}^{\mathrm{O}}=$ Joaquim Tavare $=$ Francisco Baura $=$ Maur ${ }^{\text {cio }}$. L., de Itaró= Juan S. Cabral = Joze M. Lunas = Pedro Leal = Antonio Pinto de San-martin $=$ Febro Acrios = Enrrique Martinez, Secretario geral.

Articulos adicionales á la Convencion Preliminar celebrada en este dia en el cuartel General del Ejercito Constitucional en la marjen esquerda del Cangue, entre las dos Altas Partes Contratantes Su Exelencia el General Prezidente de la Republica Rio Grandense, Su Exelencia el General en Jefe Defensor de la Constituicion de la Republica Oriental del Uruguay, Dom Fructuoso Rivera cuyas disposiciones se obligan las mismas Altas Partes Contratantes a cumplir y observar tan fiel e religiosamente como se insertas se trallaria en la referida Convension Preliminar de que hacen parte:

Articulo $1 .^{\circ}$ - El General em Jefe Defensor de la Constituicion se obliga a hacer -se elejir y proclamar por el Pueblo Oriental, en el mas curto espacio de tiempo posible Presidente de la misma Republica: empleando para lograr-lo toda su influencia y aquellos medios ọue puede emplear todo Ciudadano para merezer la confiansa y el voto Nacional.

Articulo 2. ${ }^{\circ}$ El General en Jefe Defensor de la Constitucion confiando en la eficasia de esos medios se obliga por si, por el Pueblo y Ejercito que representa a no decender jamas de la 
silla de la Presidensia en el termino marcado por la Ley, sin pasar inmediatamente a ocupar el hogar de Comandante General de Campana de la Republica Oriental con las atribuiciones hasta aqui legalmente conferidas a este empleo, y nunca menos, a fin de que pueda suceder a su turno a seo proprio sucesor en la silla de la Presidencia, cuando este disciendo de clla y asi susesivamente pasará de Presidente a Comandante General de Campana, y de Comandante General de Campana a Presidente, por todo el tiempo que durare la actual guerra de Independencia gloriosamente sustentada por el Pueblo Rio-Grandense.

Articulo $3 .^{\circ}$ - El Presidente Rio-Grandense se obliga en nombre por e de parte de su Republica a mantener con todas sus fuerzas y recursos aun cinco años despues de conquistada y reconocida la Independência del Rio Grande por El Gobierno Imperial, la influência y la preponderancia politica en el Estado Oriental del General en Jefe Defensor de la Constituicion, siempre que esta preponderancia fueren dispertadas de cualquier modo que sea por un partida, faccion o Potencia Estrangera.

Articulo $4 .^{\circ}$ - Los Articulos que anteceden se conservaran inviolablemente secretos. Para firmeza de los qual Nos, los abajo firmados Plenipotenciarios de Sus Excelencias el General Presidente de la Republica Rio Grandense, y el General en Jefe Defensor de la Constitucion de la Republica Oriental en virtud de nuestros respectivos Plenos Poderes firmamos los presentes articulos adicionales a la Convencion Preliminar con nuestros punos, y le hisimos poner el sello que usamos. Fecha en el Cuartel General del Ejercito Constitucional en la marjen esquierda del Cangué a los veinte y un dias del mez de Agosto de mil ocho sientos treinta e ocho anos = Andres Lamas $=$ Martiniano Chilavert $=$ Joze Mariano de Mattos $=$ Nos, Fructuoso Rivera, Brigadier Grãl de la Republica y Grãl en Jefe del Est ${ }^{\circ}$ Constitucional, y demas Jefes reunidos para emponerse de los articulos adicionales ala Convencion preliminar de paz, acordada entre los mismos S.S. Convencionados al efecto, y bien examinados los ratificamos como se huviera sido escritos en la misma convencion preliminar, empenando nuestra palabra y fe publica para cumplir y hacerla cumplir por todos los medios que que estea a nuestros alcanses y para mayor seguridad la firmamos, siendo refrendada por el Secretario Grãl del Est ${ }^{\circ}$ en el Cuartel Grãl de Cangué a veinte y un dia del mez de Agosto de mil ocho sientos treinta e ocho anos.= Fructuoso Rivera $=$ Felix Ed. ${ }^{\text {do }}$ Aguiar $=$ Pablo Peres $=$ Rufino Baucá $=$ Vicente Vinó $=$ Angelo Nunes $=$ Luciano Blanco $=$ Venancio Fiores $=$ Fort unato Mieres $=\mathrm{Juan}$ Santander $=$ Hipolito C. Quadros = Joze Maria Luna $=$ Joaquim Tavares $=$ Francisco Bauza $=$ Mau ${ }^{\text {cio }}$ L. de Itaro $=$ Juan J. Cabral $=$ Pedro Leale $=$ Abor. Oro $=$ Antonio Pinto de San-Martin $=$ Febro Acrios - Enrrique Martinez - Registrada a folhas quatorze o Livro primero do de Registro Secretaria de Estado dos Negocios do Exterior, Piratiny dezassete de Setembro de mil oito centos trinta e oito - João Candido de Campos. 